\title{
Hypofractionated radiotherapy results of patients with malign glioma aged 60 and over
}

\author{
@ 1 Gülçin Ertaş, @Ali Rıza Üçer, @Can Azak, @Muzaffer Bedri Altundăg \\ University of Health Sciences Dr. Abdurrahman Yurtaslan Ankara Oncology Training and Research Hospital, Department of Radiation \\ Oncology, Ankara, Turkey
}

Cite this article as: Ertaş G, Üçer AR, Azak C, Altundağ MB. Hypofractionated radiotherapy results of patients with malign glioma aged 60 and over. J Health Sci Med 2021; 4(6): 871-875.

\begin{abstract}
Aim: The aim of this study is to examine the treatment results of 25 malign glioma patients, aged $>60$ who underwent hypofractionated radiotherapy, respectively.

Material and Method: Total excision was applied to 14 of the patients, subtotal excision was applied to 5 and biopsy was applied only to 3 . Three patients were evaluated as inoperable. Pathological diagnosis is compatible with glioblastoma in 22 patients, gliosarcoma in 2 patients and anaplastic astrocytoma in 1 patient. Hypofractionated radiotherapy was applied to all patients in the dose range 2.66-3.4 Gy x 10-15 fractions. Ten patients were treated concurrent temozolomide with radiotherapy and then 4-6 cures of adjuvant temozolomide. Only concurrent temozolomide treatment was applied to 3 patients, while only adjuvant temozolomide was applied to 4 patients.

Results: The median age is 72 years. (min 60-max 86). The rate of patients with comorbid disease is $44 \%$. The median followup period of the patients is 5 months (min 1-max 22). 6-months, 1-year, 18-months overall survival were $47 \%, 20 \%, 10 \%$, respectively. Median survival in patients aged $>70$ is 3 months and 8 months in patients $<70$ ( $p=0.025$ ). Median survival is 10 months in patients receiving both concurrent and adjuvant temozolomide treatment, and median survival is 3 months $(\mathrm{p}=0.007)$ in patients who do not receive it.

Conclusion: Overall survival is statistically better in patients under the age of 70 and patients receiving both concurrent and adjuvant temozolomide therapy with hypofractionated radiotherapy.
\end{abstract}

Keywords: Malignant glioma, hypofractionated dose, chemotherapy, elderly

\section{INTRODUCTION}

High-grade malignant gliomas are $50 \%$ of all brain tumors in adults. Glioblastoma multiforme (WHO grade 4) constitutes $75 \%$ of high-grade malignant gliomas, and most of these patients are over 60 years of age (1). The prognosis for patients with glioblastoma is generally poor; age, Karnofsky performance status (KPS), type of surgery are important prognostic factors. Anaplastic gliomas (WHO grade 3, anaplastic astrocytoma, anaplastic oligoastrocytoma, anaplastic oligodendroglioma) constitute approximately 25\% of high-grade malignant gliomas in adults. In these patients, besides age, surgical method and KPS, molecular genetic factors are also important in prognosis. The standard treatment approach in patients with high-grade malignant glioma is $60 \mathrm{~Gy}$ (2 Gy x 30 fractions) adjuvant radiotherapy (RT) after surgical excision and concomitant temozolomide, followed by 6 cycles of adjuvant temozolomide. With standard treatment approaches, overall survival in these patients is $16-17$ months $(2,3)$.However, this treatment approach may cause problems due to toxic effects, especially in patients over 60 years of age and/or with poor performance status.

Various hypofractionated radiotherapy and chemotherapy approaches have come to the fore in these patients to reduce treatment-related toxicity and shorten the treatment period (4-13). The use of chemotherapy alone in this patient group has also been investigated as another treatment option. $(14,15)$.

The aim of this study is to evaluate the treatment results in patients with malignant glioma aged 60 years and older who received hypofractionated radiotherapy, respectively. 


\section{MATERIAL AND METHOD}

The study was carried out with the permission of University of Health Science Dr. Abdurrahman Yurtaslan Ankara Oncology Training and Researchs Hospital Clinical Research Ethics Committee (Date: 09.06.2021, Decision No: 2021/06-1223). All procedures were carried out in accordance with the ethical rules and the principles of the Declaration of Helsinki.

\section{Patients}

Twenty-five patients who underwent hypofractionated radiotherapy between February 2012 and December 2020 with the diagnosis of high-grade malignant glioma in our clinic were included in the study. Twenty-two patients had been operated at baseline. The operation type was gross total excision in 14 patients, subtotal excision in 5 patients, and biopsy only in 3 patients. Three patients were initially considered inoperable. Histopathological examination results were consistent with glioblastoma in 22 patients, gliosarcoma in 2 patients, and anaplastic oligoastrocytoma in 1 patient. The most common hypofractionated dose schedule was 2.66 Gy x 15 fractions (15 patients). (Table 1). In addition, 3 Gy x 15 fractions ( $\mathrm{fx}$ ) radiotherapy was applied to 7 patients, 3.4 Gy x $10 \mathrm{fx}$ to 1 patient, 3 Gy $\mathrm{x} 13 \mathrm{fx}$ to 1 patient, and $3 \mathrm{~Gy} \times 16 \mathrm{fx}$ hypofractionated radiotherapy to 1 patient. Ten patients were treated with temozolomide concomitantlywith radiotherapy and then adjuvant 4-6 courses of temozolomide. While 3 patients were treated with only temozolomide concurrently with radiotherapy, only adjuvant temozolomide therapy was applied to 4 patients. Patients were followed up with brain MRI every 3-6 months after radiotherapy.

\begin{tabular}{|c|c|c|}
\hline & No & $\%$ \\
\hline \multicolumn{3}{|l|}{ Radiotherapy } \\
\hline 2.66Gy x 15 fraction & 15 & 60 \\
\hline 3Gy x 15 fraction & 7 & 28 \\
\hline 3Gy x 13 fraction & 1 & 4 \\
\hline 3.4Gy x 10 fraction & 1 & 4 \\
\hline $3 \mathrm{~Gy}$ x 16 fraction & 1 & 4 \\
\hline \multicolumn{3}{|l|}{ Chemotherapy } \\
\hline Concomitant & 3 & 12 \\
\hline Adjuvant & 4 & 16 \\
\hline Concomitant+adjuvant & 10 & 40 \\
\hline
\end{tabular}

\section{Statistical Analysis}

SPSS version 22 was used for statistical analysis. KaplanMeier method was used in the overall survival analysis of the patients. Patient, tumor, and treatment-related variables were evaluated by univariate analysis. $\mathrm{p}<0.05$ was considered statistically significant.

\section{RESULTS}

Table 2 shows the general characteristics of the patients. Fourteen of the patients were male and 11 were female. The number of patients with comorbid disease was $11(44 \%)$, the most common comorbid disease was hypertension (45\%). Comorbid diseases other than hypertension are diabetes mellitus and cerebrovascular disease. A lower lip epidermoid carcinoma was detected in one patient as second primary cancer. The median age of the patients was 72 ( $\min 60$-max 86$)$. The average time between operation and RT is 40 days ( $\min 15-\max 75)$.

\begin{tabular}{|c|c|c|}
\hline & No & $\%$ \\
\hline \multicolumn{3}{|l|}{ Gender } \\
\hline Male & 14 & 56 \\
\hline Female & 11 & 44 \\
\hline \multicolumn{3}{|l|}{ Surgery } \\
\hline Gross total excision & 14 & 56 \\
\hline Subtotal excision & 5 & 20 \\
\hline Biopsy only & 3 & 12 \\
\hline Inoperable & 3 & 12 \\
\hline \multicolumn{3}{|l|}{ Comorbid Disease } \\
\hline Yes & 11 & 44 \\
\hline No & 14 & 56 \\
\hline \multicolumn{3}{|l|}{ Tumor Diameter } \\
\hline$\geq 5 \mathrm{~cm}$ & 9 & \\
\hline$<5 \mathrm{~cm}$ & 12 & \\
\hline
\end{tabular}

The median follow-up period is 5 months (min 1-max 22). Overall survival at 6 months, 1 year, and 18 months was $47 \%, 20 \%$, and $10 \%$, respectively (Figure 1).

In univariate analysis, the median survival was 3 months in patients aged 70 years and older, and 8 months in patients younger than 70 years $(\mathrm{p}=0.025)$ (Figure 2). Median survival was 2 months in patients receiving concomitant temozolomide therapy with RT, 1 month in patients receiving only adjuvant temozolomide therapy, and 10 months in patients receiving both concomitant and adjuvant temozolomide therapy $(\mathrm{p}=0.065)$. Median survival was 10 months in patients who received both concomitant and adjuvant temozolomide treatment, and 3 months in patients who did not receive it $(\mathrm{P}=0.007)$ (Figure 3). In addition, a significant difference was found in the Spearman correlation test between the performance status of the patients and the tolerance of concomitant and adjuvant temozolomide therapy $(\mathrm{p}=0.05)$. Median survival was 4 months in patients with a tumor size of $5 \mathrm{~cm}$ and above, and 5 months in patients with a tumor size of less than $5 \mathrm{~cm}$, and no statistical difference was found ( $\mathrm{p}=0.36)$. The median survival was 3 months in patients with an operation-radiotherapy interval of 35 days or more, and 5 months in patients with less than 35 days ( $p=0.98$ ). The prognostic significance of the IDH mutation could not be evaluated because the number of patients who were examined was insufficient. 


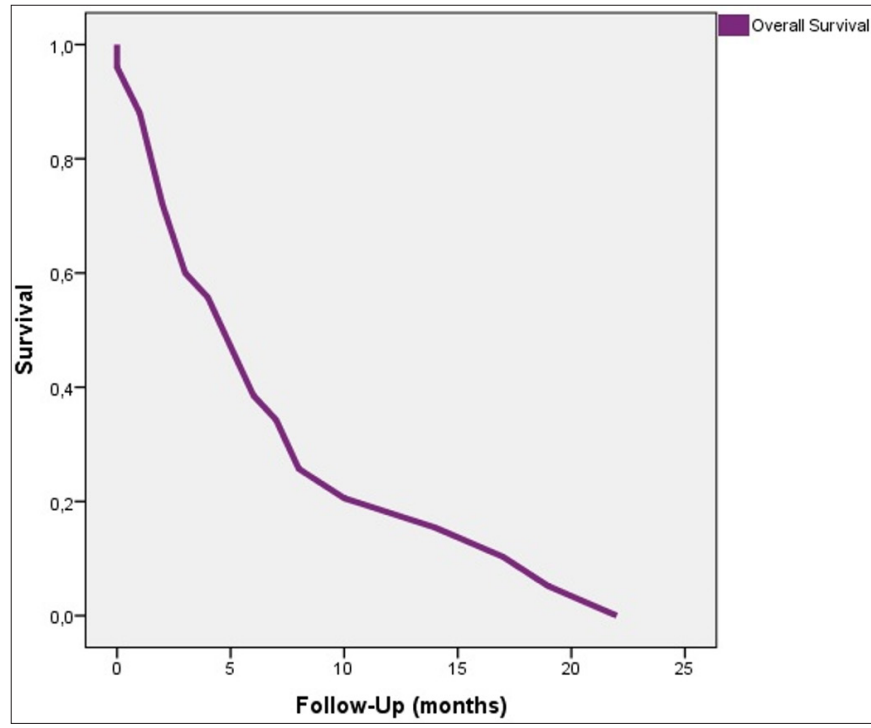

Figure 1. Overall survival of patients

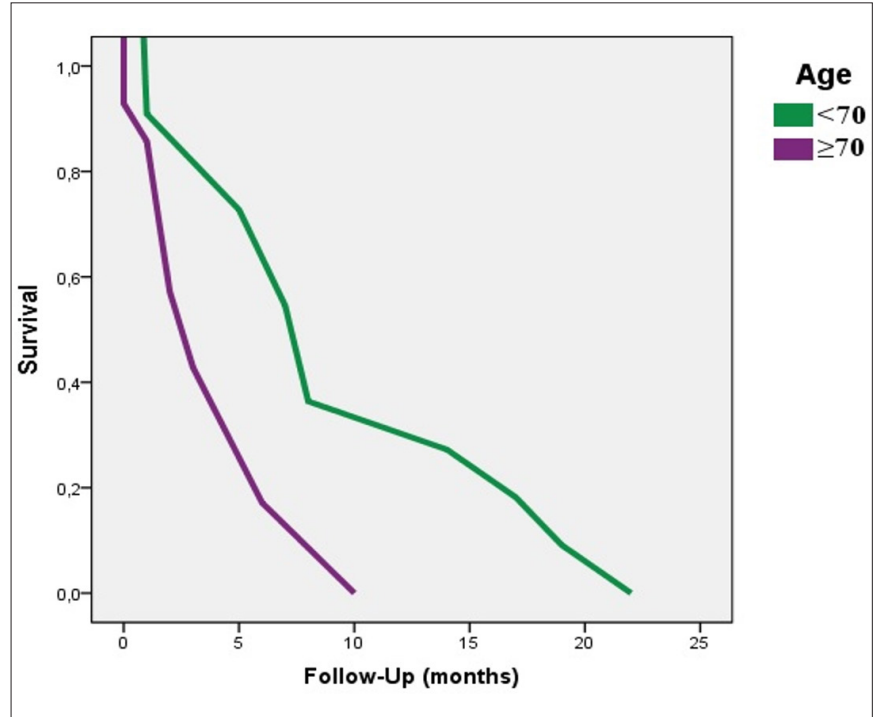

Figure 2. Survival in patients aged 70 years and older versus under 70 years of age

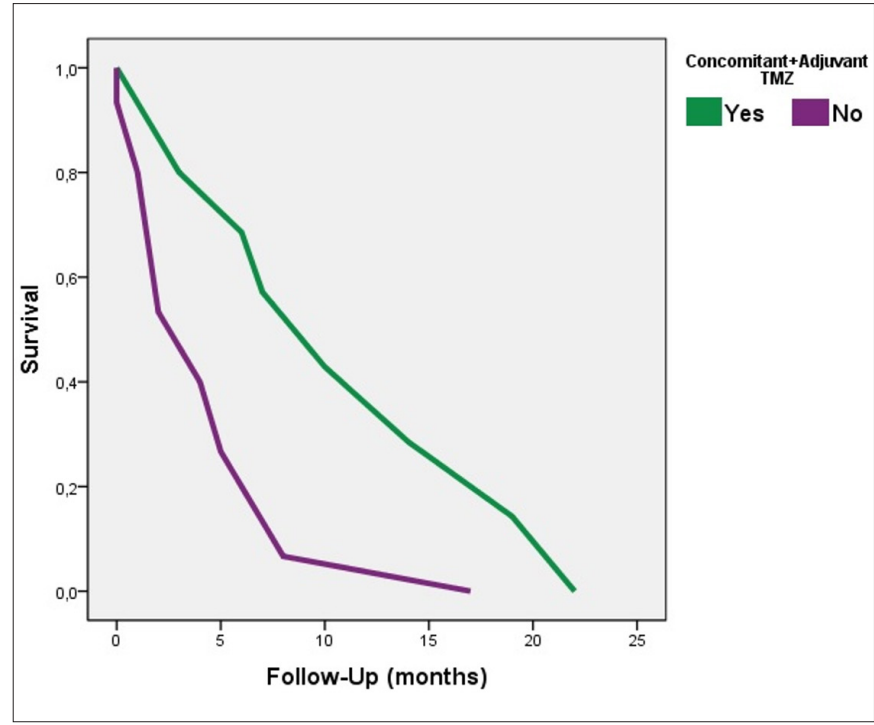

Figure 3. Survival in patients receiving concomitant and adjuvant temozolomide and patients not taking it

\section{DISCUSSION}

The standard treatment approach in patients with highgrade malignant glioma is 60 Gy adjuvant radiotherapy and concomitant temozolomide after surgical resection, followed by adjuvant temozolomide therapy. However, shorter-term hypofractionated radiotherapy options have come to the fore due to the difficulties of tolerating and completing standard treatment in patients over 65 years of age and /or with poor performance status.

In Nordic randomized phase 3 study, survival outcomes of 342 patients with glioblastoma who received the standard radiotherapy regimen, temozolomide only, and hypofractionated radiotherapy alone were compared. In this study, it was reported that the survival results were similar compared to standard radiotherapy in the patient groups treated with both temozolomide and hypofractionated radiotherapy. The median survival was shown as 8.3, 7.5 and 6 months, respectively (11).

In the study of Minniti et al. (10), the median overall survival and progression-free survival of patients receiving standard RT (60 Gy) and hypofractionated RT as well as concomitant and adjuvant temozolomide were retrospectively compared. Survival outcomes were similar in both groups (12 and 5.6 months, 12.5 and 6.7 months, respectively). In the study of Kimberly et al. (16), when the survival results of 4498 patients who received standard radiotherapy (93.4\%) and hypofractionated radiotherapy (6.6\%) were compared, it was reported that the survival results of patients who received standard treatment were better. However, in this study, the fact that chemotherapy and surgical treatment were less applicable due to the higher incidence of comorbid diseases due to the older age of the patients receiving hypofractionated treatment.

In our study, hypofractionated radiotherapy was applied to all patients $(2.66-3.4 \times 10-15 \mathrm{fx})$ in the dose range, and 10 patients were treated with temozolomide concomitantly with radiotherapy and then adjuvant 4-6 cycles. While 3 patients were treated only with temozolomide concurrently with radiotherapy, only adjuvant temozolomide therapy was applied to 4 patients. The median overall survival of patients is 5 months. However, due to the small number of patients in our study, the inclusion of patients who underwent both biopsy and inoperable in the survival analysis calculation may have caused the analysis to be performed with a heterogeneous group. This should be considered when evaluating the survival analysis results.

In the study of Perry et al. (4), treatment results of 562 patients who received concomitant- adjuvant temozolomide (50\%) treatment in addition to hypofractionated radiotherapy (2.66 Gy x $15 \mathrm{fx}$ ) and 
hypofractionated radiotherapy were compared. It was observed that survival results were better in patients who were also treated with temozolomide (median survival 9.3 months-7.6 months). In our study, $40 \%$ of the patients who received hypofractionated radiotherapy were administered concomitant and adjuvant temozolomide, and the median survival in these patients was significantly better than the patients who received only adjuvant or only concomitant temozolomide with RT. In addition to the efficacy of the treatment, this may be associated with the better performance status of the patients who can be treated with both concurrent and adjuvant temozolomide treatment compared to the patients who receive only concurrent or only adjuvant temozolomide treatment. While temozolomide treatment was ongoing, treatment-related thrombocytopenia was detected in 2 of the patients, and pulmonary thromboembolism was observed in 1 patient.

In parallel with the type of surgery performed in patients with high-grade malignant glioma, overall and disease-free survival times are prolonged in patients who underwent total excision compared to patients who underwent subtotal excision and biopsy $(17,18)$. In our study, 14 patients underwent total excision, 5 subtotal excision, and 3 patients only biopsy. Gross total excision was performed in most patients. If there is no comorbid disease that will prevent the operation, standard surgical approaches can be applied in elderly patients aged 60 and over, as in younger patients.

One study compared the treatment outcomes of patients who received combined chemoradiotherapy (89.6\%) with those who received only radiotherapy $(10.4 \%)$ in 1479 patients with high-grade malignant glioma who had only undergone biopsy for different reasons. (13). In this study, it was observed that there was a significant survival advantage in the patient group receiving chemoradiotherapy compared to the group receiving only radiotherapy (median 9.2 months-5.6 months). In our study, the number of patients who only underwent biopsy was 3 , and only radiotherapy was applied to 1 patient, simultaneous chemotherapy with radiotherapy was applied to 1 patient, and both adjuvant and concurrent chemotherapy was applied to 1 patient. The average survival of these patients is 6 months.

In our study, 3 patients were initially considered to be inoperable and were treated as high-grade malignant glioma according to clinical and radiological findings. In the treatment of these patients, only hypofractionated radiotherapy was applied to 1 patient, and concurrent and adjuvant chemotherapy with RT was applied to 1 patient. In one patient, RT followed by adjuvant temozolomide treatment was given, and the treatment was terminated after the second cycle of temozolomide due to side effects. The average survival of these 3 patients is 6 months.
In patients with high-grade malignant glioma, performance status is an important prognostic factor in addition to age. In our study, the ECOG performance score was at the median level of 2 .

\section{CONCLUSION}

Overall survival was statistically better in patients receiving both concomitant and adjuvant chemotherapy with hypofractionated radiotherapy. The better performance status of this group of patients facilitated the full administration of both radiotherapy and chemotherapy protocols. In patients under 70 years of age, overall survival is statistically better than the older group.

\section{ETHICAL DECLARATIONS}

Ethics Committee Approval: The study was carried out with the permission of University of Health Science Dr. Abdurrahman Yurtaslan Ankara Oncology Training and Research Hospital Clinical Researchs Ethics Committee (Date: 09.06.2021, Decision No: 2021/06-1223).

Informed Consent: Because the study was designed retrospectively, no written informed consent form was obtained from patients.

\section{Referee Evaluation Process: Externally peer-reviewed.}

Conflict of Interest Statement: The authors have no conflicts of interest to declare.

Financial Disclosure: The authors declared that this study has received no financial support.

Author Contributions: All of the authors declare that they have all participated in the design, execution, and analysis of the paper, and that they have approved the final version.

\section{REFERENCES}

1. Arvold ND, Reardon D.Treatment options and outcomes for glioblastoma in the elderly patients. Clin Interv Aging 2014; 9: 357-67

2. Stupp R ME, Hegi WP. Mason et al. Effects of raditherapy with concomitant adjuvan temozolomide versus radiotherapy alone on survival in glioblastoma in a randomised phase III study: 5-year analysis of the EORTC-NCIC trial. Lancet Oncol 2009; 10: 459-66

3. Scoccianti S, Magrini SM, Ricardi U, et al. Patterns of care and survival in a retrospective analysis of 1059 patients with glioblastoma multiforme treated between 2002 and 2007: a multicenter study by the Central Nervous System Study Group of Airo( italian Association of Radiation Oncology). Neurosurgery 2010; 67: 446-58

4. PerryJR, Laperrier NO, Callaghan CJ, et al. Short-course Radiation plus temozolomide in elderly patients with glioblastoma. $\mathrm{N}$ Engl J Med 2017; 376,: 1027-37.

5. Glaser SM, Dohopolski MJ, Balasubramani GK, et al. Glioblastoma multiforme (GBM) in the elderly: initial treatment strategy and overall survival. J Neurooncol 2017; 134: 107-18.

6. Kim H, Leiby BE, Shi W, et al .Too little, too soon: short course radiotherapy in elderly patients with glioblastoma. J Clin Oncol 2016; 34: 2191-2. 
7. Cao JQ, Fisher BJ, Bauman GS, et al. Hypofractioneted radiotherapy with or without concurrent temozolomide in elderly patients with glioblastoma multiforme a review of ten-years ingle instutitional experience. J Neurooncol 2012; 107: 395-405.

8. Nguyen LT, Touch S, Nehme-Schuster H, et al. Outcomes in newly diagnosed elderly glioblastoma patients after concomitant temozolomide administration and hypofractioneted radiotherapy. Cancers (Basel) 2013; 5: 1177-98.

9. Reyngold M, Lassman AB, Chan TA, et al. Abbreviated course of radiation therapy with concurrent temozolomide with high grade glioma in patients of advanced age or poor functional status.J. Neuro Oncol.2012; 110: 369-74.

10. Minniti G, Scaringi C, Gaetano L, et al. Standard (60 Gy) or short-course (40 Gy) irradiation plus concomitant and adjuvant temozolomide for elderly patients with glioblastoma: a propensity match analysis. Int J Radiat Oncol Biol Phys 2015; 1; 91: 109-15.

11. Malmstrom A, Gronberg BH, Marosi C, et al. Temozolomide versus standard 6-week radiotherapy versus hypofractionated radiotherapy in patients older than 60 years with glioblastoma: the Nordic randomised, phase 3 trial. Lancet Oncol 2012; 13 916-26.

12.Muni R, Minniti G, Lanzetta G, et al. Short-term radiotherapy followed by adjuvant chemotherapy in poor-prognosis patients with glioblastoma.Tumori 2010; 96: 60-4.

13. Kole AJ, Park HS, Neboa DY, et al. Concurrent chemoradiotherapy versus radiotherapy alone for 'biopsy-only' glioblastoma multiforme. Cancer 2016; 122: 2364-70.

14.Laigle-Donadey F, Figarella-Branger D, Chinot O, et al. Upfront temozolomide in elderly patients with glioblastoma. J Neurooncol 2010; 99: 89-94.

15. Gallego Perez-Larraya J, Ducray F, Chinot O, et al. Temozolomide in elderly patients with newly diagnosed glioblastoma and poor performance status: an ANOCEF phase II TRIAL. J Clin Oncol 2011; 29: 3050-5.

16. Mak KS, Ankit A, Qureshi MM, et al. Hypofractionated shortcourse radiotherapy in elderly patients with glioblastoma multiforme: an analysis of the National Cancer Database: Cancer Med 2017; 6: 1192-200.

17. Oszvald A, Guresir E, Setzer M, et al. Glioblastoma therapy in the elderly and the importance of the extent of resection regardless of age. J Neurosurg 2012; 116: 357-64.

18. McGirt MJ, Chaichana KL, Gathinji M, et al. Independent association of extent of resection with survival in patients with malignant brain astrocytoma. J Neurosurg 2009; 110: 156-62. 